Document de Recherche du Laboratoire d'Économie d'Orléans

Working Paper Series, Economic Research Department of the University of Orléans (LEO), France DR LEO 2019-03

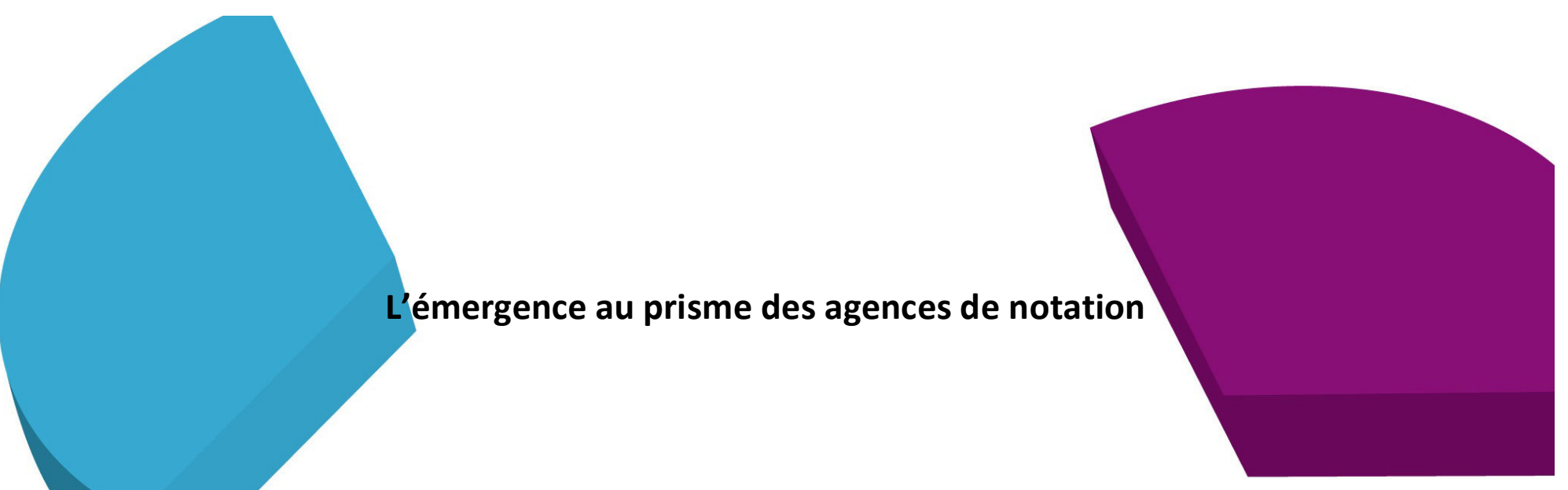

Thierry MONTALIEU

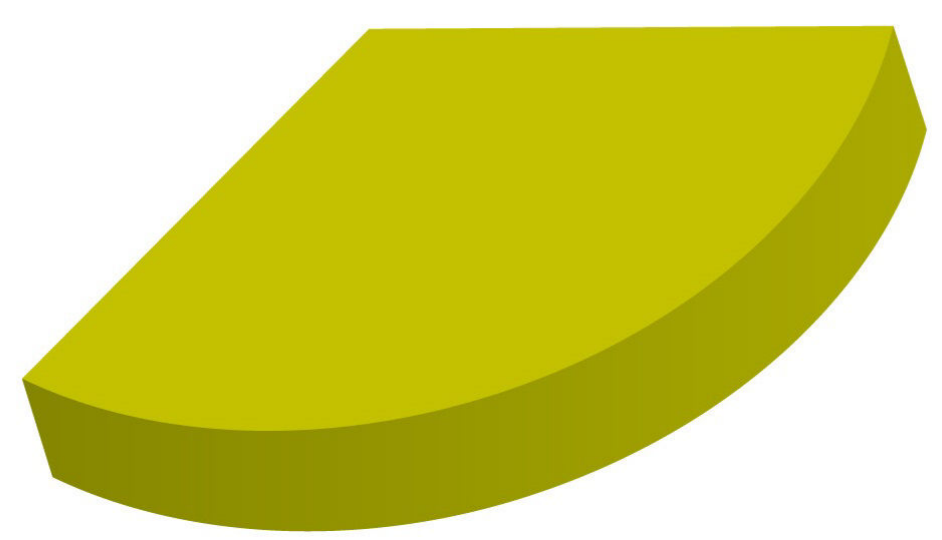




\title{
L'émergence au prisme des agences de notation
}

\author{
Thierry Montalieu ${ }^{1}$ LEO-Université Orléans
}

\section{Résumé :}

Alors que les concepts d'émergence et de risque semblent appartenir à des mondes différents, ils répondent cependant à des logiques convergentes, en particulier la volonté d'offrir aux analystes et aux décideurs des typologies à même de comparer les expériences et les politiques économiques des nations en développement. Ce constat s'est plutôt renforcé avec les épisodes récurrents de crises financières avec la mise en avant des principes de soutenabilité et de crédibilité des stratégies économiques. Des éléments statistiques descriptifs (graphiques, corrélations simples) sont utilisés pour visualiser convergences ou divergences entre mesure du niveau de développement et mesure du risque-pays et surtout repérer les cas limites ou les exceptions.

Mots-clés : pays émergents, risque-pays, notation, typologie, IDH, revenu/habitant.

\section{Abstract:}

Emerging and country-risk analyses seem to come from very different conceptual visions but respond to some logical convergences, for instance willingness to give comparisons in developing process for analysts and policymakers. This fact was reinforced with recurrent financial crises when principles as sustainability and credibility rose up in the development debate. Some basic statistics instruments (charts and simple correlation) are used to display ranking and classification and to understand possible outliers in the relationship between emergence and risk.

Keywords: emerging countries, country-risk, rating, types, HDI, income per capita.

\section{Codes JEL : O10, O57, Y10}

\footnotetext{
${ }^{1}$ Univ-Orléans, CNRS, LEO ; adresse : Laboratoire d'Economie d'Orléans, Rue de Blois - BP 26739, 45067 Orléans Cedex 2 ; e-mail : thierry.montalieu@univ-orleans.fr.
} 


\section{Introduction :}

La notion d'émergence est d'abord apparue pour décrire le processus de développement des marchés financiers dans les économies en développement. C'est évidemment au sein de l'institution internationale en charge de cette problématique, la Société Financière Internationale, que cette terminologie a connu ses premiers usages. Pour contourner le mauvais fonctionnement de l'intermédiation financière domestique (des banques ne jouant pas leur rôle efficacement) et pour sortir de la spirale de la dette commerciale externe, la mobilisation de l'épargne domestique à travers les canaux de la finance directe pouvait apparaitre alors comme un processus salutaire. D'un autre côté, la notion de risque est intimement liée au processus de développement. Dans son Rapport sur le Développement 2014, la Banque Mondiale retenait la thématique : managing risk for development.

Il est donc légitime, près de 30 ans plus tard de voir comment le concept d'émergence et les typologies qui en découlent ont été renouvelés (ou pas) à travers les informations traitées par les agences de notation et leur méthodologie de rating. Où se situent les pays émergents dans l'échelle des notations du risque-pays (économique, financier et sociopolitique) ? Existe-t-il une identité entre la typologie de l'émergence et le souci de classement du risque dans une logique de soutenabilité (dans le sens des trois composantes du risque) du développement ? Peuton parler d'une convergence des agences de rating sur l'évaluation de la situation de tels ou tels groupes de pays ? Quelle trajectoire des émergents dans les classements du risque-pays ?

Cet article cherche d'abord à développer une analyse comparative (critique) de plusieurs technologies de mesure du risque-pays ; comparaison dans le temps pour identifier les innovations et les constantes dans cette activité et comparaison dans l'espace pour apprécier les convergences et les divergences en matière de mesure du risque. Il s'agira ensuite d'évaluer la pertinence de ces mesures pour identifier des « clubs » de pays que l'on pourrait rattacher à une typologie de type "pays émergents ", "pays intermédiaires ", pays à orientation commerciale outward-looking ou inward-looking, pays à bonne qualité institutionnelle, pays à trajectoire financière soutenable, ...

Cette étude s'articule autour de quatre techniques de mesure du risque : ECR (Euromoney Institutional Investor PLC), ICRG (PRS group), COFACE et Dun \& Bradstreet. Il convient de noter à ce stade que nous ne retiendrons pas les notations issues des agences les plus diffusées (Moody's, Standard \& Poor's ou Fitch) car elles sont essentiellement centrées sur la notation financière (privée ou souveraine) et pas sur l'appréciation globale du risquepays qui recouvre un périmètre d'analyse plus large. Ces mesures permettent de classer les pays, soit sur une échelle continue de notation (sur 100 pour Euromoney et ICRG) soit à l'aide d'une échelle lettre (8 catégories A1 à E pour la COFACE et 7 groupes principaux pour D\&B de DB1 à DB7).

Alors que les deux concepts, celui d'émergence et celui de risque-pays, peuvent sembler très différents dans leur objet et leur méthode de mesure, nous montrerons dans un premier temps que leur histoire respective recèle plus de convergence que de divergence. Dans un second temps et à travers les mesures proposées par les quatre agences de notation du risque-pays présentées ci-dessus, nous verrons d'abord dans une optique transversale et par des analyses graphiques simples, si le croisement de données de risque (Euromoney, ICRG) et de mesure du développement (RNB par habitant et IDH) permet d'identifier des tendances fortes mais aussi des valeurs aberrantes. Toujours en statique, nous verrons ensuite si les "classes de risque » identifiées par ces agences (COFACE, Dun \& Bradstreet) présentent des similitudes avec les grandes typologies institutionnelles des économies (OCDE, PMA, différents « clubs » d'émergents).

\section{I - Emergence et risque-pays : un débat conceptuel}

\section{IA - $\underline{\text { Une histoire commune pour des objets différents? }}$}

Le souci de classement n'est ni une particularité des agences de notations ni une démarche réellement nouvelle. Si son objet peut sembler différent entre l'évaluation des risques pour des investisseurs, des créanciers ou de simples partenaires commerciaux, l'identification de groupes de pays éligibles à telle ou telle politique émanant des 
institutions internationales ou encore la démarche purement académique d'offrir des typologies de modèles de développement, des convergences sont néanmoins décelables.

Au regard des évolutions récentes des économies mondiales, certains ont pu croire dans un premier temps que la crise mondiale enclenchée en 2008 aboutissait à une sorte « d'inversion des rôles » (Prasad 2011) dans le sens où les économies sous-tensions ou explicitement en crise étaient plutôt des économies développées (Islande, Irlande, Grèce, Portugal) victime de la surfinanciarisation de leur économie ou d'une stratégie de change devenue peu pertinente. Mais la crise de certains BRICS ou le ralentissement de nombreuses économies émergentes ont dans un second temps rappelé que l'émergence et le risque faisaient généralement « bon ménage » (Faure et Peltier, 2013).

\section{a) des histoires parallèles}

Comme nous l'avons dit dès l'introduction, la notion d'émergence est frappée d'une tâche originelle dans la mesure où elle vient du monde de la finance et du contexte mondialisé qui l'a caractérisée pendant les deux dernières décennies du XXème siècle. Au-delà de la clause de style, ce développement conceptuel a correspondu de fait à l'essor rapide de l'activité de notation qu'il s'agisse d'abord du rating des dettes souveraines et privées (type Standard and Poor's, Moody's et Fitch) puis ensuite de l'approche plus large du risque-pays et de la qualité de l'environnement des affaires.

Certes, l'activité de notation financière nait aux Etats-Unis au XIX ̀̀me siècle pour répondre aux craintes suscitées par des épisodes de panique financière et surtout pour sécuriser le marché obligataire en plein développement dans la logique du financement des grandes infrastructures (chemin de fer, canal, ...). Les grandes agences de notation apparaissent respectivement en 1909 pour Moody's, en 1916 pour Poor's, 1922 pour Standard et enfin 1924 pour Fitch. En 1994, l'agence Dagong (Chine) est créée. Mais les analyses en termes de risque-pays voient véritablement le jour de façon embryonnaire dans les années 1960 et plus significativement à partir des années 80, à l'occasion du processus de globalisation financière et en particulier des IDE passant de 52 milliards USD en 1980 à 2272 milliards USD en 2007 (Gaillard 2015).

D'un autre côté, le groupe des pays en développement perçu jusque dans les années 80 comme globalement homogène même si des sous-groupes disposaient déjà d'une existence propre (groupe des 77 à la CNUCED dès 1964 ou le Mouvement des non-alignés fondé en 1955), va connaitre une déstructuration profonde à l'occasion de la crise de la dette de 1982 et des politiques d'ajustement structurel qui vont suivre. Ce tournant de 1982 participe à l'éclosion d'une « discipline post-moderne » mêlant nécessaire modernisation économique et mutation politique (Story 2014). Des trajectoires radicalement différentes vont se faire alors jour entre l'arrivée des NPI (de générations successives) conforme à l'archétype du Miracle Est-Asiatique (selon le célèbre rapport de la Banque Mondiale publié en 1993) et la « décennie perdue du développement » pour l'Afrique et l'Amérique latine. Depuis une dizaine d'années, la question de savoir si l'épicentre de l'économie mondiale se déplace vers les émergents a laissé place, après la crise financière, à la question de savoir si la notion de découplage entre vieilles économies industrialisées et nations émergentes était aussi pertinente que cela (CEPII 2009).

\section{b) des questionnements convergents}

L'analyse du risque-pays survient d'un questionnement qui consiste à marier différents niveaux d'analyse : projet, entreprise et pays (Nivoix 2012). Si on considère que le rendement d'un projet relève d'abord d'un calcul microéconomique (de type Valeur Actuelle Nette ou Taux Interne de Rendement, en univers certain ou probabilisable), la qualité des partenaires (entreprises clientes, fournisseurs, co-financeurs, associés) représentent déjà une échelle supplémentaire de risque potentiel. Mais le risque ultime est évidemment formé par l'environnement macroéconomique, institutionnel et sociopolitique dans lequel le projet va se dérouler pendant sa durée de vie. On le voit ici, la complexité du problème va bien au-delà de l'analyse financière d'une entreprise émettant des titres, de la solvabilité de tel ou tel opérateur (y compris public) ou même d'un potentiel défaut souverain. 
Comment se compose une bonne politique économique qui réduit le risque-pays ou une bonne politique de développement qui accompagne un pays dans un processus de modernisation et d'amélioration des performances humaines et sociétales ? Les paradigmes dominants, qu'il s'agisse d'une part du défunt Consensus de Washington (stabilisation macroéconomique ajustement structurel) ou de sa version renouvelée (croissance inclusive et bonne gouvernance) et d'autre part des OMD rebaptisés dorénavant ODD reposent largement sur un socle commun aux notions d'émergence et de risque-pays : une croissance durable soutenue par une mondialisation maitrisée (attractivité des territoires, financement de long terme, ouverture commerciale), le développement du capital humain et des institutions de qualité.

Bien entendu, cette trajectoire idéale est parfois remise en cause par des accidents...Bellocq et Zlotowski (2011) insistent par exemple sur la nécessaire mise en question de l'intégration financière mondiale dans le processus d'émergence. D'autres soulignent la myopie des marchés qui ont beaucoup de mal à distinguer les bons risques des mauvais en passant ainsi d'une contagion discriminante à une contagion pure (cf. crise asiatique de 1997, Brana et Lahet 2005). De manière plus structurelle, le difficile équilibre à trouver dans le rôle que les régulateurs (type BRI avec les Accords de Bâle) cherchent à faire jouer aux agences de notation financière souligne les dangers d'un risque systémique endogène à l'action de ces dernières (Degos et Ben Hmiden 2015).

\section{IB - Une technologie de mesure du risque qui emprunte à l'économie du développement et à la}

\section{macroéconomie internationale}

En nous fondant sur la présentation de quatre techniques de mesure du risque-pays, nous montrons que l'identification de variables pertinentes repose sur une démarche qui cherche l'exhaustivité mais qui, en même temps, pêche pas un certain manque de rigueur et de fondements analytiques comme cela a été souligné depuis longtemps (Oetzel et al. 2001, Afonso 2003, Madhu 2005 ou encore Schroeder 2008). Plus récemment des études ont souligné le manque de crédibilité de l'activité de credit-rating au moment des crises financières aussi bien dans sa capacité à alerter que dans celle d'orienter le marché une fois la crise déclenchée : Jaballah (2015) dans le cas de la crise des Subprimes et Eijffinger (2012) dans le cas de la crise des dettes souveraine dans l'Eurozone.

\section{a) un panorama de quatre mesures du risque-pays}

Au risque de caricaturer un peu la complexité de ce secteur d'activité mais à des fins pédagogiques, on peut dire que les technologies de mesure du risque-pays peuvent se classer en deux grandes catégories : celles qui établissent un score (une note généralement comprise entre 0 et 100 points) à partir de la performance d'un pays relativement à de nombreux indicateurs quantitatifs et celles qui à partir d'une échelle de notation discontinue (échelle lettres) élaborent des regroupements, a priori, homogènes correspondant à des classes de risque et en laissant une large part aux avis d'expert (dimension plus qualitative).

- Les techniques à échelle de notation continue : Euromoney Country Risk (ECR) et International Country Risk Guide (ICRG)

La mesure ECR (groupe Euromoney) développe une méthodologie avec six familles principales de variables : risque politique (pondéré à 30\% de la note totale), risque économique (30\%), risque structurel (10\%), accès au financement bancaire et aux marchés financiers (10\%), indicateurs de dette $(10 \%)$ et ratings de crédit $(10 \%)$. Le risque politique est appréhendé à travers des indicateurs tels que la corruption, le risque de non-transfert souverain, le risque institutionnel, l'environnement réglementaire et politique ; tous ces éléments permettent l'attribution de points au regard de la pondération définie préalablement. Le risque économique fait la synthèse de la stabilité bancaire, des perspectives de croissance, du taux de chômage, de la situation des finances publiques, de l'orientation de la politique monétaire et de la stabilité du change. L'évaluation structurelle repose pour sa part sur les données démographiques, la qualité des infrastructures physiques, le fonctionnement du marché du travail et la qualité du capital humain (soft infrastructure au sens de systèmes éducatif et de santé). A titre illustratif, 186 pays étaient ainsi noté en janvier 2014 allant de la Micronésie (note la plus faible à 2.00) à la Norvège (note la plus élevée à 89.79). On notera qu’en 2009 


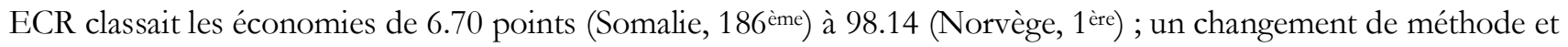
la crise financière mondiale explique la chute de 10 points observée pour le premier du classement.

La technologie ICRG (groupe PRS) retient trois grandes familles d'indicateurs : risque politique ( $50 \%$ du score final), risque économique (25\%) et risque financier (25\%). Là encore chaque famille regroupe un grand nombre de sousfacteurs faisant pour chacun l'objet d'une quantification. Pour la dimension politique du risque, ICRG observe 12 variables qui « rapportent » chacune entre 4 et 12 points pour un total de 100 (pondéré ensuite à 50 points pour la note synthétique) : stabilité du gouvernement (unité gouvernementale, pouvoir législatif, soutien populaire), conditions socioéconomiques (chômage, pauvreté, confiance des consommateurs), code d'investissement (viabilité contractuelle, rapatriement des profits, délais de paiements), conflits internes (guerres civiles, violences politiques, désordre social), conflits externes (guerres, conflits frontaliers, pressions diplomatiques), corruption, pouvoirs militaires, tensions religieuses, tensions ethniques, lois et ordre public, contrôle démocratique, qualité de l'administration. Pour la dimension économique du risque, ICRG mesure cinq composants : le produit par habitant (en dollars, méthode Atlas), la croissance économique, le taux d'inflation, le solde budgétaire et la balance courante (en $\%$ du PIB). Quant à la dimension financière, elle couvre également cinq indicateurs : Dette/PIB, Service de la dette/Exportations de biens et services, solde courant/exports, réserves de change (exprimés en mois d'importation) et enfin volatilité du taux de change (aussi bien dans sens d'une dépréciation que d'une appréciation excessive). En janvier 2014, ICRG établissait un classement pour 140 pays allant de 90,8 points pour la Norvège à 37,5 points pour la Somalie. On note immédiatement les conséquences de choix méthodologiques différents sur le spectre de la notation beaucoup plus étroit ici que dans le cas d'ECR. Ce spectre réduit a probablement des conséquences sur les rangs dans le sens où quelques écarts de points généreront des écarts de classement potentiellement plus importants. Par ailleurs, la notation ICRG est encadrée par une note basse (Worst Case Forecast) et une note haute (Best Case Forecast) à partir de projections à un et cinq ans. Cela permet de distinguer des pays dont la note absolue serait proche mais avec des profils de risque malgré tout différents.

- Les techniques à échelle de notation discontinue (COFACE, DUN \& BRADSTREET) :

Une analyse un peu critique des mesures «purement» quantitatives proposées par les deux exemples précédents conduit à envisager des approches où les contenus plus descriptifs, voire monographiques ainsi que les avis d'expert tiennent une place plus importante. La multiplication d'indicateurs quantitatifs sélectionnées de manière finalement peu analytique (s'agit-il de véritables indicateurs avancés de crise ? quels fondements théoriques ?) n'évite pas le caractère ad-hoc de certains choix (pondération, redondance, ...) et donc une forme de subjectivité que l'on illustrera dans les travaux empiriques ci-après.

La mesure COFACE établit une échelle-lettres à 8 niveaux (A1 à A4 puis B, C, D, E) et traite 160 pays (en 2018). L'analyse de la COFACE s'appuie sur trois expertises complémentaires : une approche macroéconomique permettant l'appréciation du risque-pays (vulnérabilité conjoncturelle, vulnérabilité financière de l'Etat, surendettement extérieur, risque de pénurie de devises, fragilité bancaire, fragilité politique), une notation spécifique de l'environnement des affaires (qualité de l'environnement institutionnel, protection des investisseurs et disponibilités des informations financières dans l'esprit de Doing Business) et une base de données retraçant 60 ans d'expérience en matière de comportement de paiements des entreprises (échelle microéconomique). La notation du risque-pays est ici le fruit d'une appréciation globale à partir de quelques données statistiques mais surtout d'une expertise qualitative d'un réseau d'experts entretenus par l'institution. En mars 2018, la répartition des pays par classes est la suivante : 6 pays A1 (risque très faible), 19 pays A2, 12 pays A3, 18 pays A4, 27 pays $B, 40$ pays $C, 23$ pays $\mathrm{D}$ et enfin 14 pays $\mathrm{E}$ (risque extrême). Pour compléter, il est intéressant d'ajouter que l'analyse est dynamisée par l'ajout d'une mention " pays sous surveillance positive/négative » pour des économies sur une trajectoire d'amélioration ou au contraire de détérioration de leur performance.

D’une manière assez proche, le cabinet Dun \& Bradstreet publie également une typologie des risques selon une échelle principale à 7 niveaux (DB1à DB7) possiblement subdivisés en quartiles pour les 6 premiers niveaux (hors 
DB7 donc). Là-aussi, à partir de données statistiques compilées mais surtout de l'expérience d'un réseau d'experts, chaque économie est analysée autour de 4 axes majeurs : risque politique (sécurité interne et externe, cohérence politique, compétences administratives), risque commercial (respect des contrats, qualité du système juridique, transparence réglementaire, corruption), risque macroéconomique (inflation, équilibre budgétaire, politique monétaire, soutenabilité de la croissance) et risque externe (balance courante, flux financiers, réserves de change, dette extérieure). Pour compléter cette première étape, D\&B y ajoute 9 catégories d'analyses plus fines : trajectoire économique de court-terme (2 à 8 trimestres), le potentiel économique de long terme (scenarii à 5/15 ans), les opportunités ou risques de marché (barrières vs incitations), les risques de change, le risque de non-transfert, la qualité de l'environnement des affaires, les chocs exogènes disruptifs (climat, séisme, dégradation des infrastructures,...), l'insécurité et les désordres civils et enfin les expressions extrêmes de la souveraineté des Etats (expropriation, nationalisation répudiation de la dette). En mars 2018, la répartition des économies selon la technologie D\&B donne le résultat suivant : 3 pays DB1 (risque le plus faible), 22 pays DB2, 16 pays DB3, 32 pays DB4, 25 pays DB5, 27 pays DB6 et 6 pays DB7.

La critique essentielle que l'on peut adresser à ces approches en termes de « clubs » est de générer des discontinuités (effet de seuil) sans que l'on comprenne toujours exactement pourquoi telle ou telle économie bascule dans une classe de risque supérieure ou inférieure. Là-encore, les illustrations empiriques qui suivent marqueront des différences pas clairement explicables. La dimension subjective de l'avis d'expert et le caractère intrinsèquement plus multidimensionnel de ces technologies confine parfois au syndrome de la « boite noire $»^{2}$.

b) des fondements académiques communs

En reprenant la typologie de Borio et Packer (2004), l'analyse du risque-pays a d'abord été restreinte à sa dimension financière à travers les trois inspirations académiques suivantes cherchant à évaluer le risque de défaut ou de nontransfert :

- L'intolérance à la dette : théorie proposée par Reinhart, Rogoff et Savastano et qui désigne l'incapacité de nombreuses économies émergentes à faire face à « des niveaux d'endettement global qui sembleraient tout à fait gérables selon les critères des pays industrialisés avancés ». (Ibid p. 48).

- Le concept de péché originel, caractéristique intrinsèque pour la plupart des pays (pouvant au premier abord être confondu avec celui d'intolérance à la dette) : «l'incapacité d'un pays à emprunter à l'étranger dans sa propre monnaie » et, par extension, à se couvrir vis-à-vis des non-résidents (Eichengreen, Hausmann et Panizza). Une telle situation accroittrait la fragilité d'un pays, car les dépréciations de change pèsent sur le service de sa dette extérieure. (Ibid p. 49)

- L'asymétrie de devises proche du concept de péché originel : elle peut se définir (Goldstein et Turner) comme « la vulnérabilité de la situation nette ou de la valeur actualisée du revenu net aux variations de change ». (Ibid p. 49)

Bien entendu, les éléments présentés ci-dessus amènent rapidement à l'idée que pour éviter la survenance d'un risque strictement financier, il convient de disposer d'un bon cadre macroéconomique et structurel et qu'il faut, pour cela, mener les bonnes politiques économiques (stabilisation de la dépense, politique monétaire, stratégie de change, insertion commerciale, qualité des infrastructures, ...) ce qui revient à inclure la dimension économique dans le risque. Cette seconde étape est en accord avec ce qu'il est convenu d'appeler le «nouveau consensus de Washington»

\footnotetext{
${ }^{2} \mathrm{Au}$-delà des controverses conceptuelles, les mesures du risque font également l'objet de raffinements techniques qui tentent au moins de gommer les critiques sur leur rusticité statistique. Voir par exemple Agliardi et al. (2012).
} 
et conduit naturellement à s'interroger sur la soutenabilité des réformes et la qualité de l'environnement sociopolitique (bonne gouvernance, stabilité) et donc d'élargir l'analyse du risque vers sa dimension politique ${ }^{3}$.

Pour relativiser cette apparente solidité théorique du cadre d'analyse du risque macroéconomique et des recommandations de politique économique qui en découle, nous pouvons nous associer aux critiques formulées par Hibou et Samuel (2011) que l'on peut résumer autour de la notion d'économie politique de la normalisation. Beaucoup des critères quantitatifs et des cibles retenus dans l'évaluation des politiques économiques relèvent davantage de conventions et de compromis que de calibrages fondés sur des analyses scientifiquement éprouvées. Ce processus normatif est probablement plus important que les objectifs ciblés eux-mêmes car il est interactif entre toutes les parties prenantes : bailleurs, organisations internationales, coopération bilatérale, Etats récipiendaires et agences de notation ajouterons-nous.

\section{II - Emergence et risque-pays : une analyse empirique}

En analyse transversale, l'objectif des techniques de mesure du risque-pays est de classer les pays les uns par rapport aux autres, soit sur une échelle de notation continue (note de 0 à 100 points pour Euromoney et ICRG) soit sur échelle lettre (catégorisation en 8 sous-groupes pour la COFACE et en 7 sous-groupes principaux pour Dun \& Bradstreet).

On peut ainsi imaginer deux approches complémentaires. La première consistera à corréler les rangs (et les valeurs) des mesures du risque (ECR, ICRG) avec les mesures habituelles de développement (Revenu par habitant et IDH)4. La seconde cherchera à mettre en relation les « clubs» formés par la typologie des échelles de notation discontinue (COFACE, Dun \& Bradstreet) d'une part et celle des regroupements institutionnels (OCDE, Emergents, PMA).

\section{IIA - Comparaison des rangs et des valeurs}

\section{a) appréciation du degré de convergence entre les mesures concurrentes du développement et du risque}

Avant de présenter quelques analyses graphiques pour repérer les écarts de mesure entre d'une part le risque (ECR et ICRG) et d'autre part le niveau de développement (RNB/habitant en dollars courants et IDH), nous pouvons d'abord offrir un panorama sur le degré de convergence des différentes mesures du risque ${ }^{5}$.

De manière assez surprenante, les mesures du risque mises en relation témoignent d'une corrélation plutôt médiocre (respectivement 0,75 et 0,72 pour les rangs et les valeurs) si on garde à l'esprit qu'elles sont censées décrire strictement le même phénomène. Il y aurait finalement plus de différences méthodologiques dans la construction des indicateurs du rating-pays que dans les approches concurrentes mesurant le développement ${ }^{6}$. Pour entrer dans le détail, on observe sur le graphique A (cf. annexe) un certain nombre de cas limites. Ainsi la France, l'Estonie la Slovénie et la Turquie présentent un rang nettement plus défavorable avec ICRG qu'avec ECR (respectivement 59e/22e $, 65^{\mathrm{e}} / 25^{\mathrm{e}}, 85^{\mathrm{e}} / 42^{\mathrm{e}}$ et $124^{\mathrm{e}} / 46^{\mathrm{e}}$ ) alors que Brunei, la République Dominicaine, l'Argentine et Cuba sont caractérisés par un profil opposé (3e/58e, 64e/99e, 81e/122e et 82e/136e).

La comparaison des valeurs (graphique $\mathrm{C}$ en annexe) et non des rangs dresse un tableau assez proche ; autrement dit, les écarts de rang ne masquent pas une forte concentration des notations. Sur une échelle de notation strictement comparable (0 à 100), la performance de chaque pays en matière de risque est globalement convergente. Cependant,

\footnotetext{
${ }^{3}$ La publication annuelle de la Banque Mondiale, Doing Business est une illustration remarquable de cette tendance à multiplier les angles d'analyse pour apprécier la qualité de l'environnement institutionnelle à même d'assurer une insertion durable dans la mondialisation et une pérennité des réformes.

${ }^{4}$ L'objectif n'est pas ici de discuter de la pertinence ou des limites des différentes mesures de développement. Nous retenons les deux mesures les plus communes malgré leurs insuffisances bien connues.

${ }^{5}$ Pour cette première analyse, les données retenues sont celles de janvier 2014 pour les mesures du risque.

${ }^{6}$ Nous avons mis en annexe deux graphiques (B et D) pour illustrer ce propos que nous ne commentons pas dans le texte car assez périphérique par rapport au cœur de notre sujet.
} 
on note que la Guinée, la Turquie, l'Estonie et la France sont relativement mieux notés par ECR que par ICRG (assez nettement au-dessus de la courbe de régression) alors que dans le même temps la Corée du Nord, Haïti, Cuba et Brunei sont sensiblement mieux évalués par ICRG.

Pour ce qui concerne la relation développement-risque, on note que les corrélations s'établissent entre 0,57 (IDH/ICRG) et 0,68 (RNB/ECR) pour les valeurs et 0,61 (IDH/ICRG) et 0,70 (RNB/ECR) pour les rangs. Il semble que la technologie ICRG soit globalement moins bien corrélée avec les mesures de développement que ne le réalise la technologie ECR. La méthodologie ICRG présente une forte pondération de la dimension politique du risque $(50 \%$ de la note) pouvant expliquer cela. Néanmoins, la relation risque-développement parait globalement établie. On trouve évidemment une écrasante majorité de pays développés parmi les meilleures notes (ou rang) du risque et réciproquement pour les pays à faible niveau de développement.

Au-delà de cette relation globale, il est intéressant d'observer les points qui échappent à ces tendances générales (points « aberrants » ou cas limites) aussi bien dans le sens d'une sous-estimation potentielle du risque que dans celui d'une surestimation possible.

\section{b) Identification des cas limites dans la relation émergence-risque : analyse graphique (approche par le rang)}

Le graphique 1 compare le rang des variables RNB/ECR ; quelques cas limites sont ainsi repérés. Certains pays bénéficient d'un meilleur rang en termes de risque ECR que ne le laisserait supposer leur classement en termes de produit par habitant. C'est le cas du Chili, du Pérou de l'Inde et du Malawi (respectivement 15e/47e, 38e/75e,

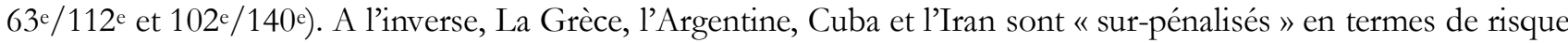

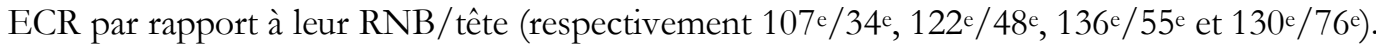

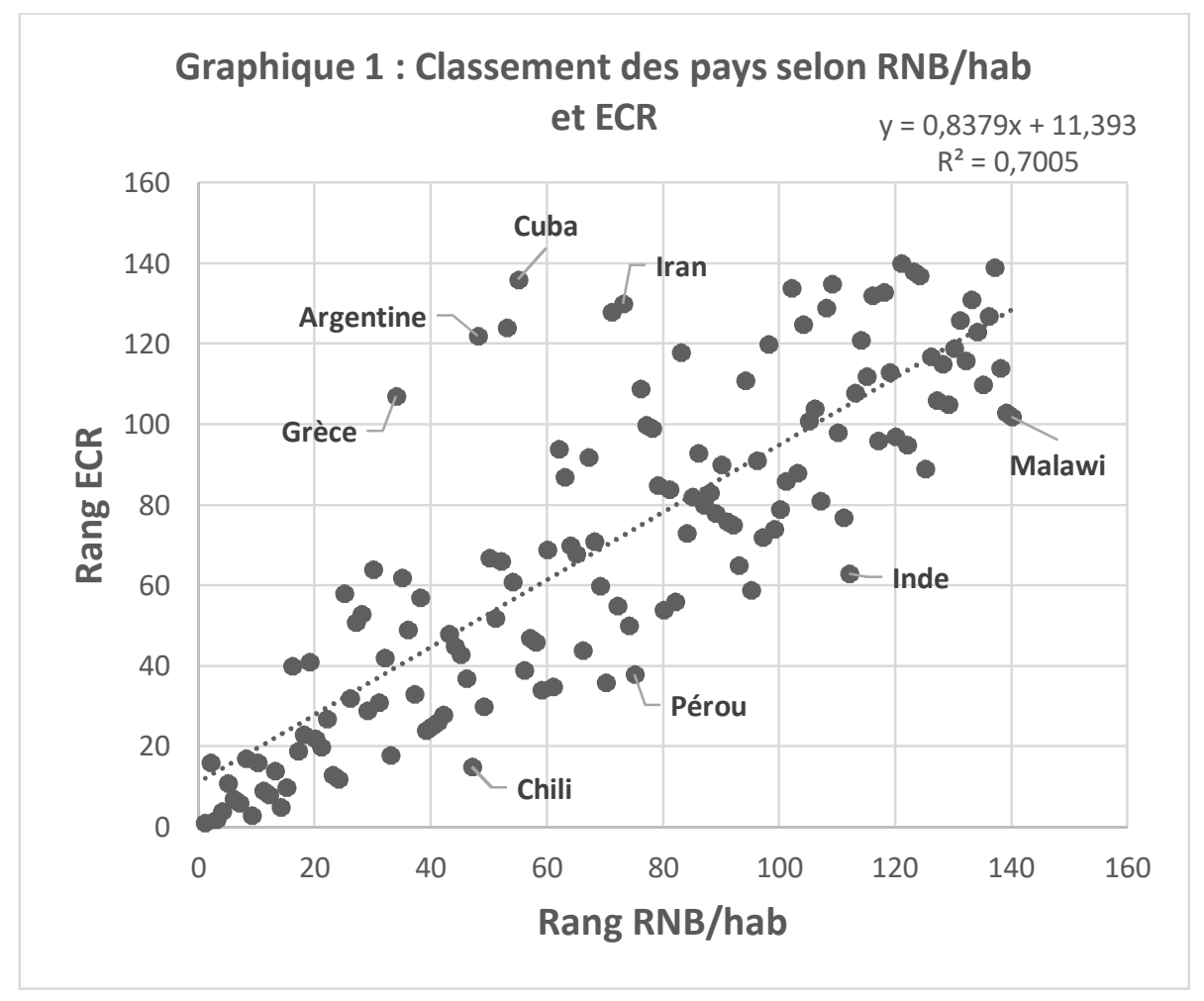

Il s'agit maintenant d'apprécier la convergence entre les rangs du couple développement-risque mesurée par les variables RNB/ICRG (graphique 2) ; le constat est différent par rapport à la comparaison précédente. L'autre mesure du risque (ICRG) produit des outliers différents : Brunei, le Botswana, les Philippines et la Bolivie sont mieux appréciés en termes de risque qu'en termes de développement (respectivement $33^{\mathrm{e}} / 25^{\mathrm{e}}, 26^{\mathrm{e}} / 72^{\mathrm{e}}, 50^{\mathrm{e}} / 95^{\mathrm{e}}$ et $51^{\mathrm{e}} / 101^{\mathrm{e}}$ ). Dans le même temps, la France, Chypre, le Venezuela et la Syrie apparaissent comme « sur-risqués » au regard de 
leur $\mathrm{RNB} /$ tête (respectivement, 59e/20e, 94e/30e, 132e/53e et 139e/102e). On observera que la mesure du risquepays par ICRG englobe davantage de variables quantifiées (plus d'avis d'experts chez ECR) et que la pondération du risque politique est plus importante (50\% de la note finale contre $30 \%$ pour ECR).

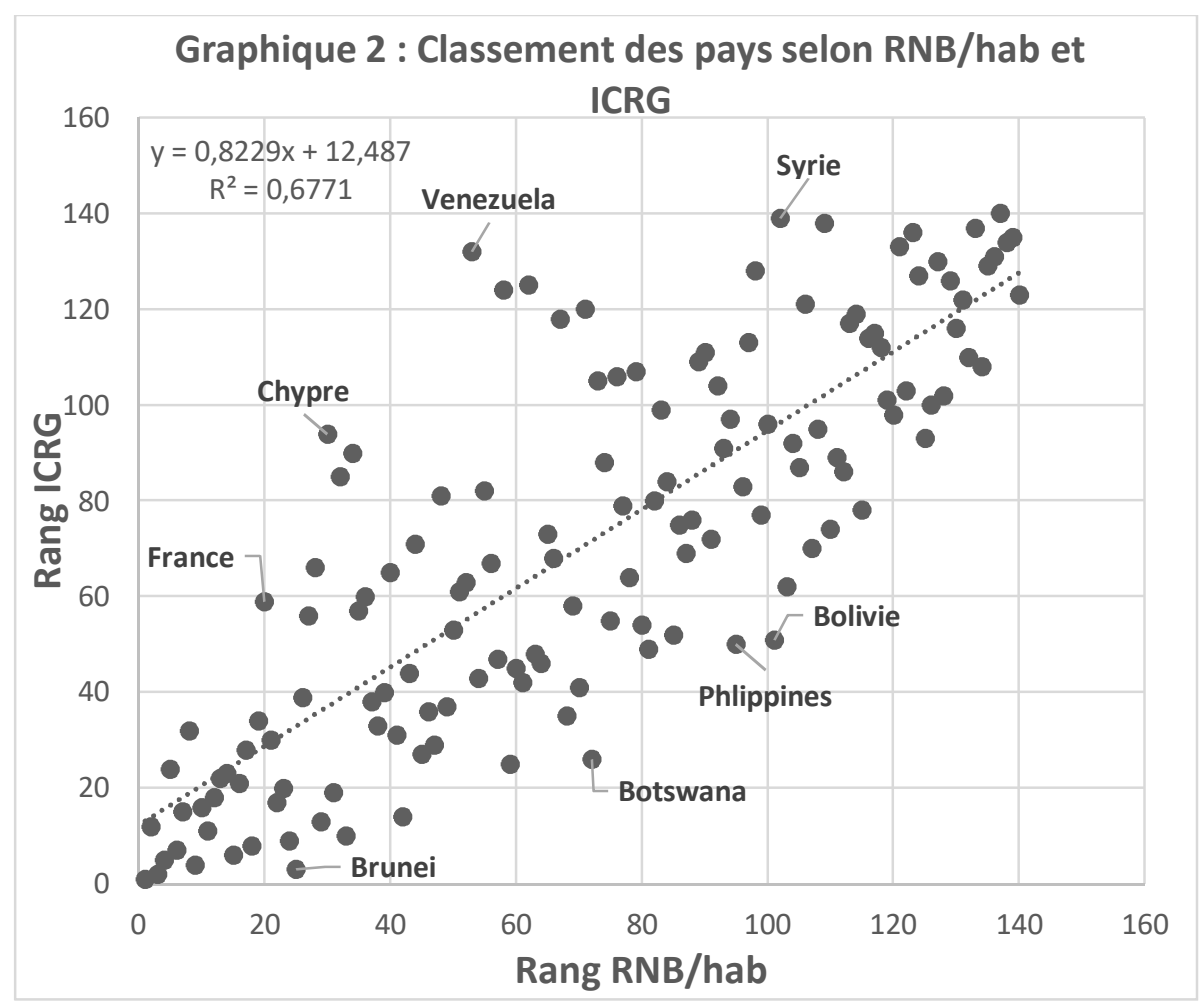

La question qu'il convient dorénavant de traiter est de capter la sensibilité de la relation développement-risque à la prise en compte d'une nouvelle mesure du développement qui est celle de l'IDH. Les comparaisons de rang IDH/ECR sont présentées dans le graphique 3 et celles concernant le couple IDH/ICRG dans le graphique 4.

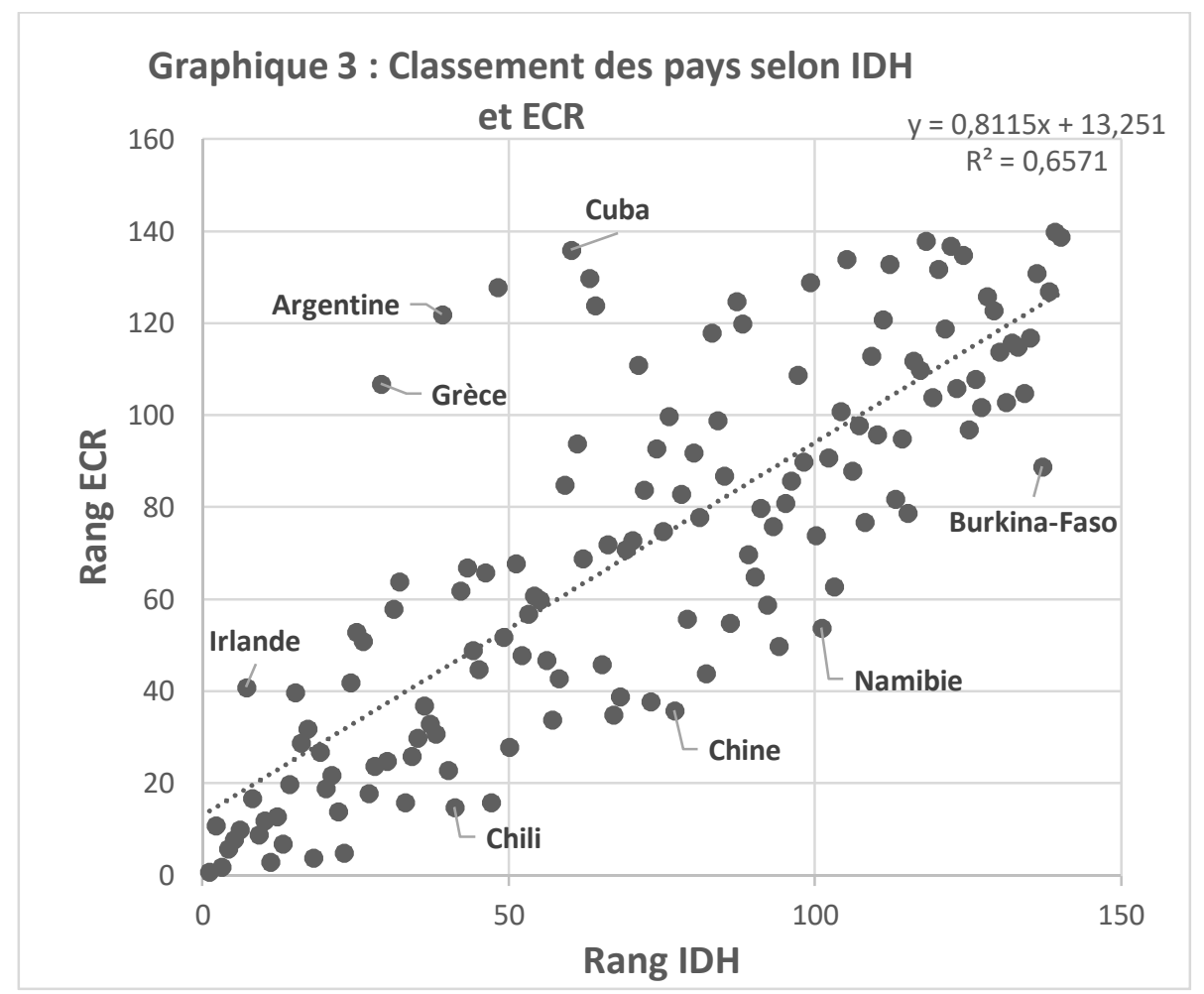


L'approche par le développement humain comme variable à mettre en relation avec les mesures de risque (ECR et ICRG) modifie sensiblement le panorama précédent ; on note l'apparition de nouveaux pays s'écartant sensiblement de la tendance générale : la Chine, la Namibie, le Burkina-Faso, Oman, et l'Angola comme pays sous-risqués par rapport à leur classement IDH et à l'opposé l'Irlande, la Slovénie ou le Belarus. La France et le Venezuela sont, pour leur part, invariants comme pays sur-risqués pour ICRG quelle que soit la mesure de développement de même que le Botswana comme pays sous-risqué. Pour ECR, les invariants sont la Grèce, l'Argentine et Cuba comme pays surévalués en termes de risque et le Chili comme pays sous-évalué en termes de risque.

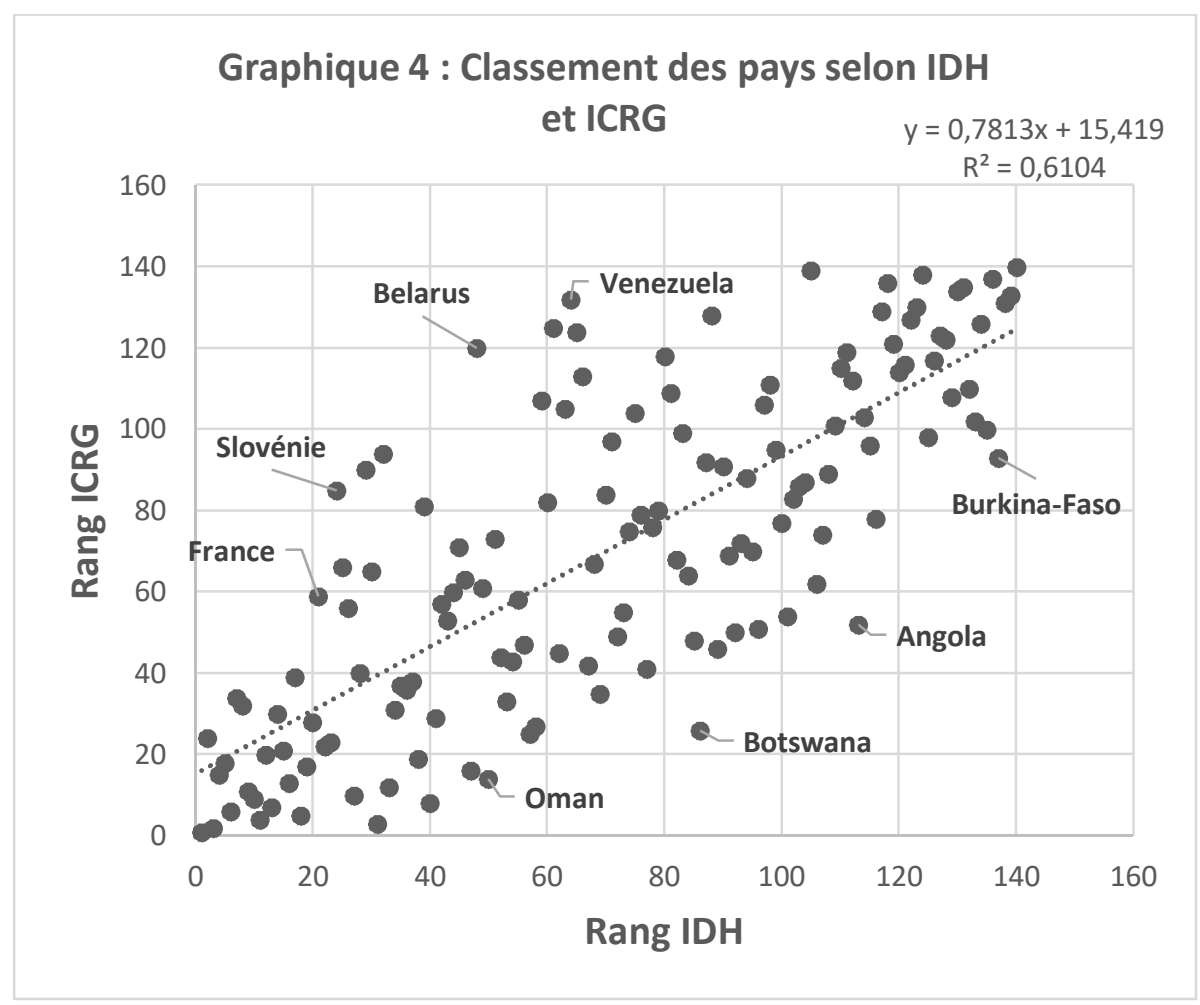

c) Identification des cas limites dans la relation émergence-risque : analyse graphique (approche par les valeurs)

Le passage d'une corrélation de rangs à un exercice similaire sur les valeurs des indices est-il de nature à modifier la description statistique de la relation émergence-risque ? La question est légitime dans la mesure où des classements comparables peuvent en fait révéler des écarts de notation sensibles d'une technique de mesure à l'autre. On peut en effet imaginer soit une forte concentration des notes ou au contraire une dispersion pour un classement donné et donc des rangs comparables. Cette analyse commence par le couple RNB/ECR (graphique 5) et le couple RNB/ICRG (graphique 6).

L'observation des valeurs (et non des rangs) sur la relation émergence-risque ne modifie pas fondamentalement le panorama dressé lors de l'approche par les rangs. Seule l'Islande apparait comme nouveau pays moins bien noté par ECR que ne le laisserait penser son revenu par habitant. Dans l'autre sens, Singapour bénéficie d'une « surcôte » en termes de notation aussi bien pour ECR que pour ICRG. Notons la situation paradoxale de Brunei qui passe d'une sur-notation chez ICRG (graphique 6) à une sous-notation de sa performance chez ECR (graphique 5). 

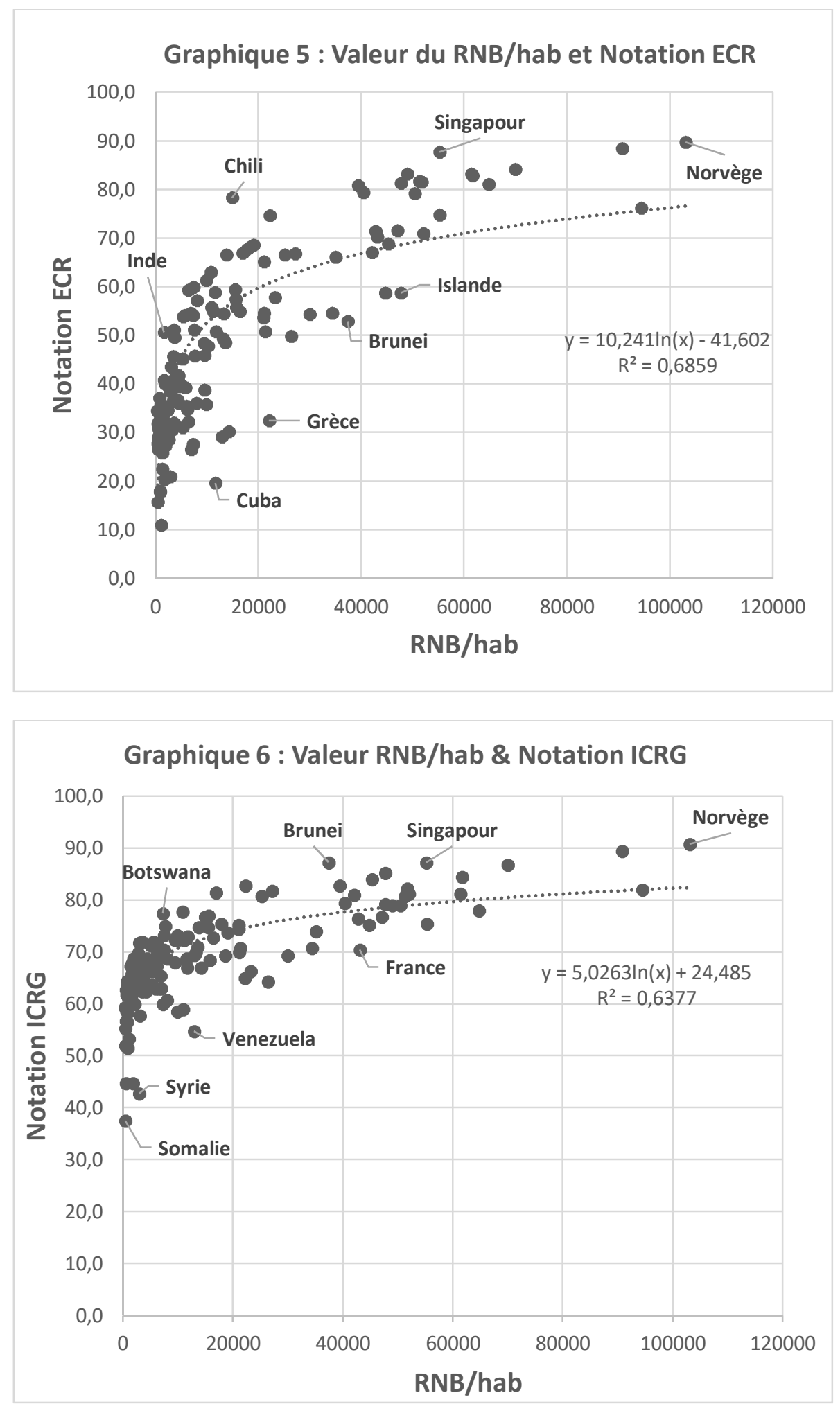

Le passage aux couples IDH/ECR (graphique 7) et IDH/ICRG (graphique 8) n'introduit pas de modifications majeures dans l'analyse. Un groupe de pays africains à niveau d'IDH faible (Sierra Leone, Burkina-Faso) et moyen (Angola, Namibie) bénéficie d'une notation (ECR ou ICRG) supérieure à ce que leur développement humain pourrait laisser supposer. 

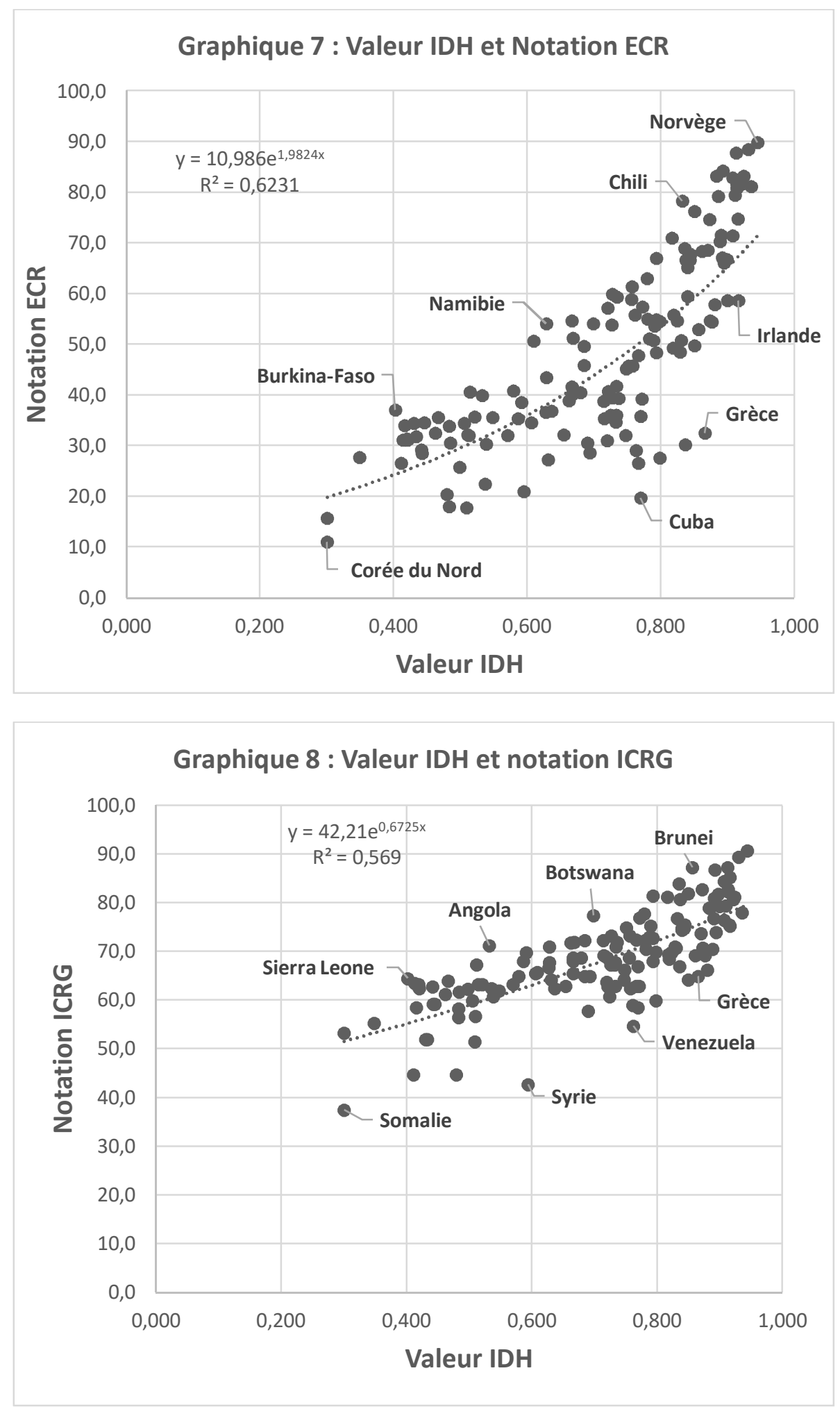

Cet ensemble de paradoxes ou de déconnexions entre les différentes mesures du risque et de l'émergence doit conduire tout naturellement à une réflexion de fond sur les conditions d'exercice des agences de notation. D'aucuns ont déjà souligné depuis longtemps la profonde incapacité de ces notations à anticiper réellement les phases de retournement par leurs techniques parfois rudimentaires qui mettent en cause leur fiabilité (Sommerville 
et Taffler 2001). D'autres ont évoqué l'idée que ces agences étaient à la fois « juges et parties » dans l'activité financière tantôt simple révélateur de la performance économique ou tantôt régulateur privé du système financier ${ }^{7}$.

\section{IIB - Comparaison des classes de risque et des « clubs » de développement}

Nous présentons dans le tableau ci-dessous (tableau 1), la double typologie du risque (131 pays doublement évalués par COFACE et Dun \& Bradstreet) et les regroupements institutionnels les plus usités dans les institutions internationales et la communauté financière (Emergents, PMA, OCDE) ${ }^{8}$. Les nombreux acronymes décrivant les générations successives des pays émergents ont déjà fait l'objet de critiques éclairantes (Dazanio 2013) qui portent en particulier sur le manque de fondements économiques à ces regroupements. Il est aussi symptomatique d'observer qu'une bonne partie de ces « clubs » a été introduite par les banques ou les agences de rating elles-mêmes (Lafargue 2011). Pour ce qui concerne cette étude, nous cherchons à croiser les classes de risque avec les différents « clubs » de pays afin d'en repérer les fondamentaux mais surtout les cas limites : pays appartenant à plusieurs « clubs », pays dont le risque semble surestimé par rapport au club d'appartenance ou au contraire sous-estimé.

En colonne, nous reprenons les classes de risque D\&B (7 classes) et en ligne, celles de la COFACE (8 classes). Puis nous utilisons un code couleur pour illustrer les clubs d'appartenance'. Ainsi, une majorité de pays entre dans une lecture diagonale plutôt attendue : faible risque pour les deux agences pour une majorité de pays OCDE (en noir gras), risque intermédiaire pour une majorité de pays émergents (en gris gras) et enfin des risques élevés pour les PMA (en bleu gras souligné). Bien entendu, l'analyse la plus intéressante est de regarder spécifiquement les cas qui échappent à cette relation globale.

a) les pays-frontières en termes de clubs

Quelques pays appartiennent à plusieurs clubs institutionnels, soit qu'il s'agisse d'émergents ayant intégré très tôt l'OCDE soit qu'il s'agisse de PMA perçus comme à fort potentiel d'émergence. On repère ainsi les deux groupes suivants $^{10}$ : OCDE/EMERGENTS avec Corée du Sud (A2/DB2), Mexique (B/DB3), Turquie (B/DB4) et EMERGENTS/PMA : Tanzanie (C/DB4), Bangladesh (C/DB5), Ethiopie (C/DB6), Zambie (D/DB5). On observera que cette double appartenance tend à faire bénéficier à ces pays d'une forme de « prime » en termes de risque puisque leur évaluation se rapproche davantage de la moyenne obtenue par le club « supérieur » en termes de développement.

\section{b) les pays sur-risqués (au regard de leur typologie d'émergence)}

Les pays OCDE sur-risqués sont pour l'essentiel des pays pour lesquels la crise financière de 2009 a laissé des stigmates durables concernant soient leur dette publique ou la dette privée (risque sur le système bancaire dans le cas de la Hongrie par exemple) : Grèce (B/DB5), Hongrie, Italie, Portugal (A3/DB4).

On observe également des émergents sur-risqués (noté au-delà du mode par au moins une agence) : Nigéria (D/DB6), Iran (E/DB5), Pakistan (C/DB6), Egypte (B/DB6), Russie (B/DB6), Argentine (B/DB5), Sri Lanka (B/DB5), Vietnam (B/DB5), Arabie Saoudite (C/DB3), Afrique du Sud (C/DB4). Cette dernière liste comprend plutôt des pays (majoritairement à niveau de développement intermédiaire) où le risque politique est perçu comme une dimension très importante au regard des performances économiques.

\section{c) les pays sous-risqués (au regard de leur typologie d'émergence)}

Moins nombreux que les précédents, quelques cas de pays sont remarquables car l'appréciation du risque est meilleure que ce pourrait laisser penser leur « club » d'appartenance.

\footnotetext{
${ }^{7}$ Voir en particulier, Mathis et al. (2009) et White (2010).

8 Pour ces différentes typologies, c'est la situation en janvier 2018 qui est retenue.

${ }^{9}$ Les pays classés pour leur niveau de risque mais qui n'appartiennent à aucun « club » sont en italique

${ }^{10}$ Pays en petites majuscules dans le tableau 1.
} 
Les émergents sous-risqués (noté au-dessous du mode par au moins une agence) : Malaisie (A4/DB3). Depuis la crise asiatique de 1997, ce pays est généralement apprécié des instances internationales (par exemple FMI) et donc des milieux d'affaires par sa capacité à assurer une bonne crédibilité de sa stratégie économique.

Quelques PMA peuvent apparaitre comme sous-risqués : Sénégal (B/DB4), Ouganda (C/DB5), Cambodge (C/DB5). Il est plus difficile de trouver une ligne de force dans cet ensemble. Concernant le Sénégal, on peut considérer qu’il bénéficie depuis de nombreuses années de l'image du « bon élève » de l'Afrique de l'Ouest auprès des institutions multilatérales et des agences de développement, ce qui peut rejaillir évidemment sur l'appréciation des investisseurs internationaux et des agences de notation.

Tableau 1 : croisement des données en termes de classes de risque et de « clubs » de développement

\begin{tabular}{|c|c|c|c|c|c|c|c|}
\hline & $\begin{array}{c}\text { DUN \& } \\
\text { BRADSREET }\end{array}$ & & & & & & \\
\hline COFACE & DB1 & DB2 & DB3 & DB4 & DB5 & DB6 & DB7 \\
\hline A1 & $\begin{array}{l}\text { Allemagne } \\
\text { Norvège } \\
\text { Suède }\end{array}$ & $\begin{array}{l}\text { Autriche } \\
\text { Luxembourg } \\
\text { Pays-Bas } \\
\text { Suisse }\end{array}$ & & & & & \\
\hline A2 & & $\begin{array}{l}\text { Etats-Unis } \\
\text { Australie } \\
\text { COREE Du SUD } \\
\text { Hong-Kong } \\
\text { Japon } \\
\text { Nelle Zélande } \\
\text { Singapour } \\
\text { Taïwan } \\
\text { Belgique } \\
\text { Danemark } \\
\text { Estonie } \\
\text { Finlande } \\
\text { France } \\
\text { Malte } \\
\text { Tchéquie }\end{array}$ & $\begin{array}{l}\text { Espagne } \\
\text { Islande } \\
\text { Israël }\end{array}$ & & & & \\
\hline A3 & & $\begin{array}{l}\text { Canada } \\
\text { Royaume-Uni } \\
\text { Slovaquie }\end{array}$ & $\begin{array}{l}\text { Chili } \\
\text { Irlande } \\
\text { Lettonie } \\
\text { Lituanie } \\
\text { Pologne } \\
\text { Slovénie }\end{array}$ & $\begin{array}{l}\text { Hongrie } \\
\text { Italie } \\
\text { Portugal } \\
\text { Koweit }\end{array}$ & & & \\
\hline A4 & & & $\begin{array}{l}\text { Botswana } \\
\text { Maurice } \\
\text { Panama } \\
\text { Malaisie } \\
\text { Qatar }\end{array}$ & $\begin{array}{l}\text { Kenya } \\
\text { Maroc } \\
\text { Colombie } \\
\text { Costa Rica } \\
\text { Pérou } \\
\text { Uruguay } \\
\text { Inde } \\
\text { Indonésie } \\
\text { Philippines } \\
\text { Thaillande } \\
\text { Bulgarie } \\
\text { Roumanie } \\
\text { E.A.U. }\end{array}$ & Chypre & & \\
\hline
\end{tabular}




\begin{tabular}{|c|c|c|c|c|c|}
\hline B & Mexique & $\begin{array}{l}\begin{array}{l}\text { Namibie } \\
\text { Sénégal }\end{array} \\
\text { Brésil } \\
\text { Rép. Domi. } \\
\text { Chine } \\
\text { Croatie } \\
\text { Macédoine } \\
\text { Serbie } \\
\text { TuRquIE }\end{array}$ & $\begin{array}{l}\text { Côte d'Ivoire } \\
\text { Tunisie } \\
\text { Argentine } \\
\text { Jamaïque } \\
\text { Sri Lanka } \\
\text { Vietnam } \\
\text { Géorgie } \\
\text { Kazakhstan } \\
\text { Grèce }\end{array}$ & $\begin{array}{l}\text { Egypte } \\
\text { Ghana } \\
\text { Russie }\end{array}$ & \\
\hline $\mathrm{C}$ & $\begin{array}{l}\text { Arabie } \\
\text { Saoudite }\end{array}$ & $\begin{array}{l}\text { Afrique du } \\
\text { Sud } \\
\text { TANZANIE } \\
\text { Trinité- } \\
\text { Tobago } \\
\text { Albanie } \\
\text { Bahrein } \\
\text { Oman }\end{array}$ & 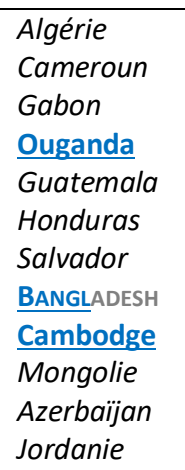 & $\begin{array}{l}\text { ETHIOPIE } \\
\text { Bolivie } \\
\text { Equateur } \\
\text { Nicaragua } \\
\text { Paraguay } \\
\text { Pakistan } \\
\text { Ouzbékistan } \\
\text { Ukraine } \\
\text { Belarus } \\
\text { Bosnie } \\
\text { Liban }\end{array}$ & \\
\hline $\mathrm{D}$ & & & ZAMBIE & $\begin{array}{l}\frac{\text { Angola }}{\text { Congo RD }} \\
\text { Malawi } \\
\text { Nigéria } \\
\text { Sierra Leone } \\
\text { Myanmar } \\
\text { Népal } \\
\text { Kirghizistan } \\
\text { Tadjikistan } \\
\text { Turkménistan }\end{array}$ & \\
\hline $\mathrm{E}$ & & & $\begin{array}{l}\text { Mozambique } \\
\text { Iran }\end{array}$ & $\begin{array}{l}\text { Soudan } \\
\text { Cuba } \\
\text { Venezuela }\end{array}$ & $\begin{array}{l}\text { Libye } \\
\text { Zimbabwe } \\
\text { Afghanistan } \\
\text { Irak } \\
\text { Syrie } \\
\text { Yémen }\end{array}$ \\
\hline
\end{tabular}

OCDE, PMA, BRICS-PPICS-CIVETS-MINT'MIST'B-BENIVM-Next Eleven

\section{Conclusion :}

Dans cette recherche à caractère exploratoire, nous nous sommes attachés à croiser les analyses en termes d'émergence (ou de niveau de développement) avec celles qui cherchent à révéler le risque-pays associé aux opérations commerciales et d'investissement à l'échelle internationale. Sur un plan purement empirique, nous avons illustré par une série de graphiques que ces deux concepts présentent d'importantes tendances communes. Mais le plus intéressant est finalement d'étudier les cas limites ou les pays-frontières. Pourquoi certains pays émergents semblent plus risqués que d'autres ? Pourquoi une même classe de risques regroupe des pays en situation parfois différente en matière de développement économique ? Parmi les explications que l'on peut retenir, la dimension politique (mesurée par les agences ou qualitativement appréciée par les institutions internationales) et un effet mémoire (pays au cœur d'importantes crises politiques ou financières passées) sont de nature à expliquer ces "points aberrants » au sens statistique du terme. Dans le premier cas, la forte pondération des variables décrivant le climat politique ou plus généralement la capacité de certains Etats à entretenir des relations pérennes et confiantes avec les grandes institutions multilatérales (une forme de soft power qui ne serait pas l'apanage des nations dominantes) pourraient introduire une déformation des distributions entre émergence et risque. Dans le second cas, des évènements historiques pourraient introduire une persistance voire un effet d'hystérèse dans les analyses qualitatives ou les avis d'expert surpondérant alors les chocs financiers ou politiques négatifs passés quand bien même des signes 
d'amélioration économique sont tangibles. Ces déconnexions ont-elles un caractère transitoire ou permanent ? Plus généralement, nous pourrions reprendre à notre compte la formule retenue par Didier et Weil (2012) pour décrire la difficulté inhérente à cette activité de mesure du risque, le complexe de Jonas : une prophétie « condamnée à être démentie lorsqu'elle est entendue, et réalisée lorsqu'elle est tue » ${ }^{11}$. Les techniques de mesure du risque se doivent d'être suffisamment originales pour apporter une valeur ajoutée dans un contexte concurrentiel mais en même temps se situer dans une forme de convergence pour assurer une crédibilité.

Au-delà de l'effort de catégorisation, une autre approche, à venir, consisterait à apprécier les trajectoires d'économies émergentes et des risques associés (approche dynamique plutôt que statique). Commet s'opèrent les changements dans les classements continus (rang et valeur) à travers les gains ou les pertes de performance ? Les améliorations de performance en termes de développement (revenu par habitant, IDH) s'accompagnent-elles systématiquement par une diminution du risque dans le temps (ECR, ICRG) ? De même, le passage d'un club à un autre en termes de développement (typologie institutionnelle) signifie-t-il forcément un changement de statut dans les échelles de notation discontinue du risque?

\section{Bibliographie :}

AFONSO A. (2003) Understanding the determinants of Sovereign Debt Rating: Evidence for the two Leading Agencies, Journal of Economics and Finance, vol. 27(1), 56-74.

AGLIARDI E., AGLIARDI R., PINAR M., STENGOS T., TOPALOGLOU N. (2012) A new country risk index for emerging markets: A stochastic dominance approach, Journal of Empirical Finance, Vol. 19, 741-761.

BELLOCQ F.X., ZLOTOWSKI Y. (2011) Les pays émergents dans l'après-crise : l'intégration financière en question ?, Revue d'Economie Financière, n¹01, 273-288.

BORIO C. \& F. PACKER (2004) Risque-Pays : évaluation des nouvelles approches, Rapport trimestriel BRI, décembre, 47-65.

BOUDES T. (2013) Présentation de l'ouvrage Les dessous du triple A - agences de notation : récit de l'intérieur, Annales des Mines - Gérer et comprendre, $\mathrm{n}^{\circ} 111,75-78$.

BRANA S., LAHET D. (2005) La propagation des crises financières dans les pays émergents : la contagion est-elle discriminante ?, Economie Internationale, $\mathrm{n}^{\circ} 103,73-96$.

CEPII (2009) Onze questions sur les grandes économies émergentes, sous la coordination de J.J. Boillot, Le Club, 149 p.

COFACE (2013) Panorama : les transformations du risque pays émergents, Les Publications Economiques de la COFACE, printemps, $12 \mathrm{p}$.

COFACE (2015) Panorama : baromètre risque pays, Les Publications Economiques de la COFACE, septembre, $18 \mathrm{p}$.

DAZIANO L. (2013) La Nowvelle Vague des Emergents, Fondapol, 44 p.

DEGOS J.G., BEN HMIDEN O. (2015) Le rôle et le pouvoir des agences de notation ont-ils changé au fil des accords de Bâle I, Bâle II et Bâle III ?, Revue d'Economie Financière, n¹20, 239-254.

DIDIER S., WEILL N. (2012) Les dessous du triple A, Agences de notation : récit de l'intérieur, Omniscience, 188 p.

DUN \& BRADSTREET (2012) Global Outlook, A D and B Special Report, July, 10 p.

EIJFFINGER Sylvester W.C. (2012) Rating Agencies: Role and Influence of Their Sovereign Credit Risk Assessment in the Eurozone, Journal of Common Market Studies, Volume 50 (6), 912-921.

FAURE F. \& C. PELTIER (2013) Pays émergents : le retour du risque pays ?, Conjoncture, novembre, BNP-Paribas, 25-35.

GAILLARD N. (2015) Le concept de risque-pays, Politique Etrangère, $\mathrm{n}^{\circ} 15-2,161-172$.

HIBOU B., SAMUEL B. (2011), La macroéconomie par le bas, Politique Africaine, n¹24, 5-27.

JABALLAH J. (2015) Impact of the Subprimes Crisis on the Reputation of Rating Agencies, Finance, Vol.36, n³ , 53-83.

LAFARGUE F. (2011) Des économies émergentes aux puissances émergentes, Questions Internationales, n $51,101-108$.

MADHU V. (2005) The Determinants of Country Risk Analysis: an empirical approach, Journal of Management Research, vol. 5(1), $20-31$.

${ }^{11}$ Repris par Boudès (2013) dans sa présentation de l'ouvrage de Didier et Weil dans les Annales des Mines. 
MATHIS J., MCANDREWS J. \& J-C. ROCHET (2009) Rating the Raters: Are Reputation Concerns Powerful Enough to Discipline Rating Agencies?, Journal of Monetary Economics, Vol. 56, 657-674.

NIVOIX S. (2012), L'évolution de l'expertise dans l'analyse du risque-pays, Prospective et Stratégie, vol. 1, n²-3, $105-124$.

OETZEL J.M., BETTIS R.A. \& M. ZENNER (2001) Country Risk Measures: How Risky are they?, Journal of World Business, vol. 36(2), 128-145.

PRASAD E.S. (2011) Inversion des rôles, Finances \& Développement, décembre, 26-29.

SCHROEDER S.K. (2008) The Underpinnings of Country Risk Assessment, Journal of Economic Survey, vol. 22(3), 498-535.

SOMMERVILLE R.A., TAFFLER R.J. (2001) LDC Credit-Risk Forecasting and Banker Judgment, Journal of Business Finance \& Accounting, Vol. 28 (3 \& 4), 447-464.

STORY J. (2014) L’analyse du risque pays : plus qu’une discipline postmoderne, Politique Etrangère, nº14-2, 11-24.

WHITE L.J. (2010) Markets: The Credit Rating Agencies, Journal of Economic Perspectives, Vol. 24, 211-226.

WORLD BANK (2013) World Development Report 2014: Risk and opportunity, managing risk for development, The World Bank Group, $362 \mathrm{p}$.

WORLD BANK (2016) Doing Business 2017: equal opportunity for all, The world Bank Group, Washington, 346 p. 


\section{ANNEXES : GRAPHIQUES COMPLEMENTAIRES}
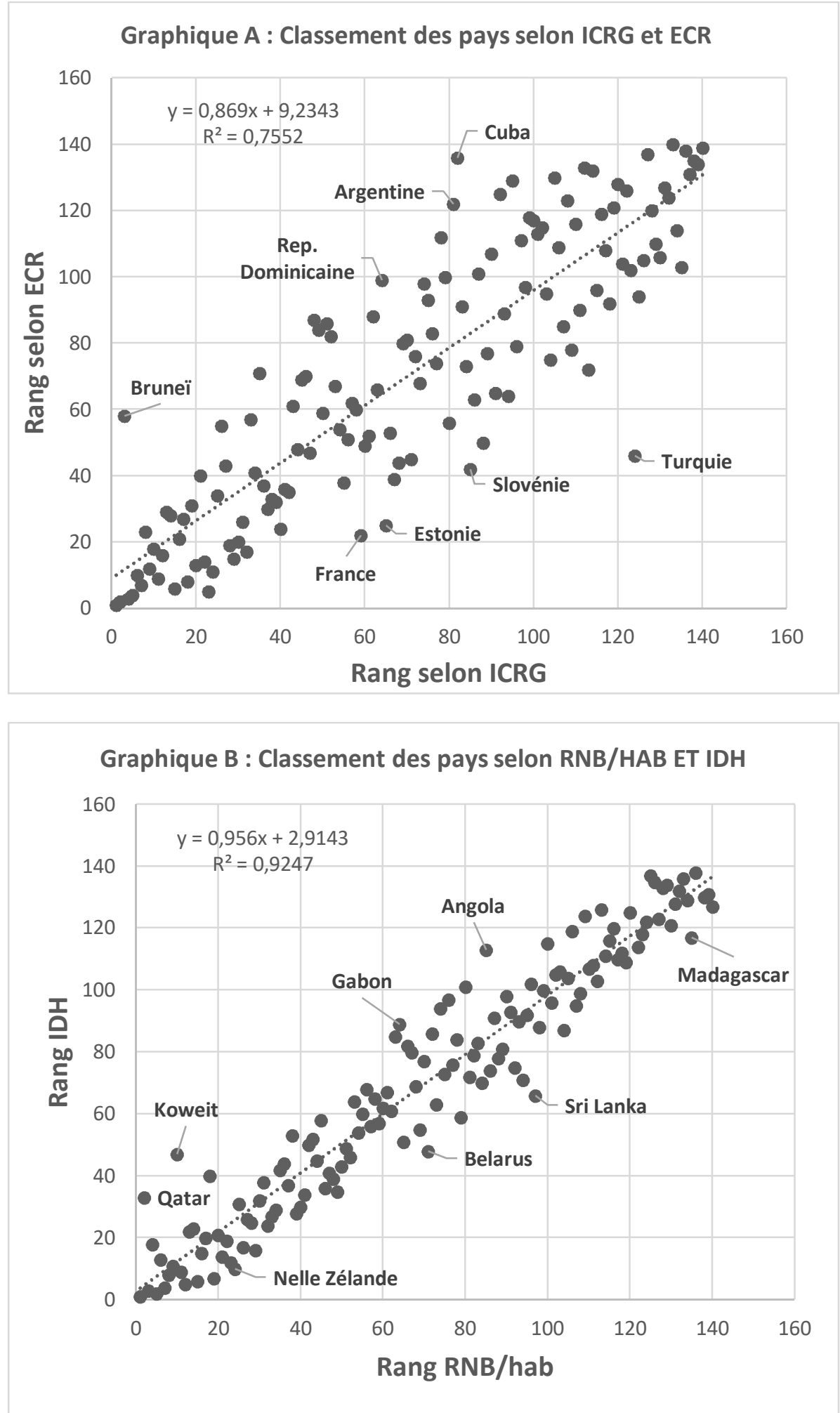

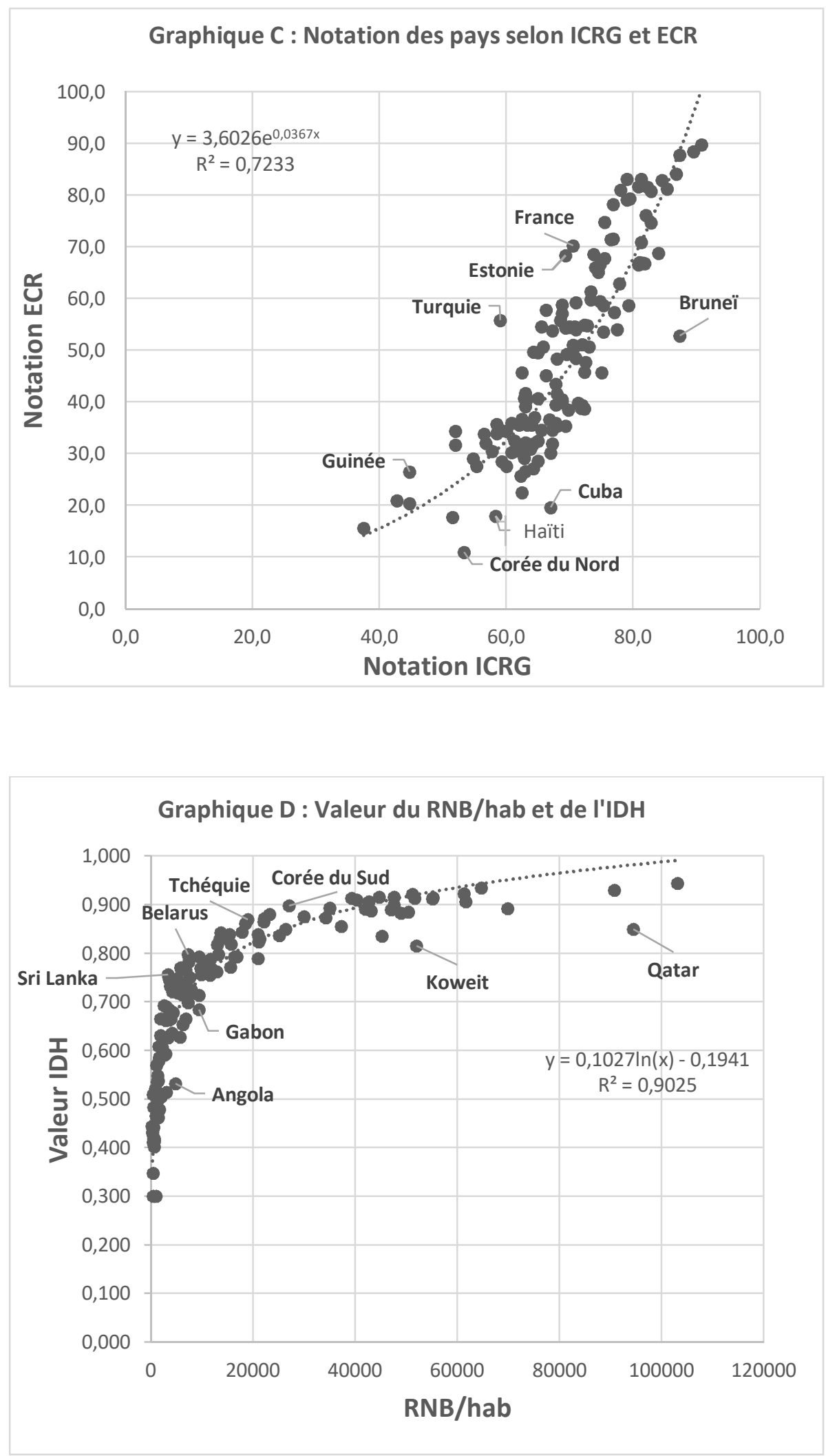